\title{
Increasing contribution of non-alcoholic fatty liver disease to hepatocellular carcinoma incidence and mortality in U.S. Medicare
}

\author{
Carrie R. Wong ${ }^{1,2}$, Joseph K. Lim $^{3}$ \\ ${ }^{1}$ Vatche and Tamar Manoukian Division of Digestive Diseases, David Geffen School of Medicine at the University of California, Los Angeles, CA, \\ USA; ${ }^{2}$ Department of Health Policy and Management, Jonathan and Karin Fielding School of Public Health at the University of California, Los \\ Angeles, CA, USA; ${ }^{3}$ Yale Liver Center and Section of Digestive Diseases, Yale University School of Medicine, New Haven, CT, USA \\ Correspondence to: Joseph K. Lim. Yale Liver Center and Section of Digestive Diseases, Yale University School of Medicine, New Haven, CT, USA. \\ Email: joseph.lim@yale.edu. \\ Comment on: Hester D, Golabi P, Paik J, et al. Among Medicare patients with hepatocellular carcinoma, non-alcoholic fatty liver disease is the most \\ common etiology and cause of mortality. J Clin Gastroenterol 2019. [Epub ahead of print].
}

Submitted Mar 25, 2019. Accepted for publication Apr 09, 2019.

doi: 10.21037/hbsn.2019.04.06

View this article at: http://dx.doi.org/10.21037/hbsn.2019.04.06

Hepatocellular carcinoma (HCC) has one of the fastest growing rates of cancer-related incidence and mortality in the United States (U.S.) (1-3). From 2009 to 2013, the average annual percent change (AAPC) for liver cancer incidence became the highest among other reported cancers for both males $(+2.9 \%)$ and females $(+3.8 \%)(1)$. In addition, cancer-related mortality rates for liver cancer are the highest among solid malignancies from 2010 to 2014 for males $(+2.6 \%)$ and females $(+3.0 \%)$ (1). Morbidity and mortality from HCC are expected to increase, predominantly driven by an increasing prevalence of $\mathrm{HCV}$-associated cirrhosis and non-alcoholic fatty liver disease (NAFLD) (2).

Similarly, morbidity and mortality from chronic liver disease (CLD) among older persons is expected to grow, with reported mortality rates confirmed to be highest among individuals older than 65 years old in the U.S. (80.4 deaths per 100,000 males, 44.4 deaths per 100,000 females in 2016) (4). Older age represents an established risk factor for HCC, with a 15 -fold increase in risk of HCV-related HCC among individuals age 65 years and older (5), which in turn is associated with increased economic impact of HCC among Medicare beneficiaries $(1,6)$.

In a recent issue of fournal of Clinical Gastroenterology, Hester et al. describe recent trends in HCC-related mortality and health care utilization among 13,648 Medicare beneficiaries from 2005 to 2014 (7). Inpatient and outpatient Medicare data were used to assess differences in 1 -year mortality and health care utilization (based on annual number of claims, total charges, and payments). The crosssectional study provided a unique snapshot of HCC and CLD trends in a large sample of Medicare beneficiaries, both those age 65 years and older, but also younger enrollees who represented $23.8 \%$ of the sample, which is larger than the estimated $17.2 \%$ of Medicare beneficiaries who are eligible for Medicare due to disability and/or endstage renal disease (ESRD) (6). Although the total number of HCC cases was greater among individuals age 65 years or older versus younger enrollees (74.6\% vs. 25.4\%), the annual percent change increase in HCC incidence was notably higher among younger enrollees (7.2 HCC cases per 100,000 for age $<65$ years $v s$. 1.7 HCC cases per 100,000 for age $65-74$ years vs. 2.7 HCC cases per 100,000 for age $\geq 75$ years). The authors' observation of a declining average age of Medicare recipients at time of incident HCC supports emerging data which suggest a disproportionate increase in HCC incidence among younger individuals, for which further investigation is needed to clarify underlying etiologic factors, which may be driven in part by incident HCC among non-cirrhotic patients with NAFLD or HBV (8).

Hester et al. additionally report important observations regarding ethnic differences in HCC etiology among Medicare recipients, with NAFLD most common among White and Hispanic enrollees, HCV most common among Black enrollees, and HBV among Asian enrollees. Although the rising impact of HCV on HCC incidence was observed 
across all ethnic groups, this trend was most striking among Black enrollees, in whom HCV-related HCC incidence increased $12.2 \%$ per year. Due to the time period studied in this analysis (2005 to 2014), the impact of all-oral direct acting antiviral (DAA) regimens for $\mathrm{HCV}$ infection was not likely reflected in these data, although ongoing differences in receipt of DAA therapy between Black and non-Black patients may contribute to the disparity in $\mathrm{HCV}$-related HCC. A nationwide Veterans Administration cohort revealed that Black patients were $21 \%$ less likely than non-Black patients to receive DAA, similar to differences observed in treatment access in the interferon era (9). NAFLD was the most common etiology of HCC in the overall sample, but the increase in HCC incidence during the study period was most profound among Hispanic enrollees, with an APC of NAFLD-HCC of 15.6 between 2008 and 2014. Underlying mechanisms for ethnic differences in NAFLD-HCC remain poorly described, but may stem in part from metabolic syndrome and underlying genetic susceptibility from polymorphisms such as PNPLA3 (patatin-like phospholipase domain containing protein 3$)(10,11)$.

As expected, 1-year mortality among Medicare recipients was higher among inpatients than outpatients in the sample ( $64.43 \%$ vs. $40.69 \%$, which likely reflect fundamental differences in baseline patient and disease characteristics. Compared with the outpatient sample, inpatient Medicare patients who developed incident HCC had a higher proportion of cirrhosis ( $54.51 \%$ vs. $24.98 \%$ ) and medical comorbidities based on Charlson comorbidity index (CCI $0.04 \pm 3.11$ vs. 3.78 \pm 2.36 ). Multivariable logistic regression in both the inpatient and outpatient samples revealed that cirrhosis and older age were associated with 1-year mortality; NAFLD (OR 1.87) was additionally associated with 1 -year mortality within the outpatient sample. However, this model was limited by the absence of key clinical variables which may influence mortality, including HCC stage at time of diagnosis, and HCC treatment. In particular, this analysis did not account for curative interventions such as liver transplantation. Based on Scientific Registry of Transplant Recipients (SRTS) data, $17.0 \%$ of patients listed for liver transplantation in the U.S. 2002 to 2017 had a listing diagnosis of HCC, with NASH representing the fastest growing HCC etiology (12).

Furthermore, Hester et al. report a significant increase in health care cost expenditure among Medicare patients with HCC from 2004 to 2013, with the highest average charges observed among patients with HBV-related HCC $(\$ 145,176)$ versus NAFLD-related HCC $(\$ 76,995)$.
Previous Medicare reports suggest that increases in both inpatient and outpatient charges may stem largely from temporal increases in medical care prices, increases in patient volume, and shifts of services from physician offices to higher-cost hospital-based outpatient settings (13). Importantly, this study by Hester et al. was limited by assessment of HCC treatment, and therefore the lower average charges among patients with NAFLD-related HCC vs. HBV-related HCC may be attributable in part due to lower eligibility for therapeutic HCC intervention. Prior studies have shown that patients with NAFLD-related HCC have more cardiac and other comorbidities than patients with other etiologies $(14,15)$ and are less likely to receive curative treatment (14). Furthermore, this model was unable to fully account for attribution of medical care utilization for management of HCC versus the underlying chronic liver disease (CLD), recognizing important differences in health care expenditure in patients with CLD and either compensated or decompensated cirrhosis. Although HCV-related HCC is predominantly observed in patients with advanced liver disease or cirrhosis, HCC is more commonly observed in patients without advanced liver disease in those with HBV or NAFLD etiology, which may further influence measures of health expenditure (14).

In short, this study by Hester et al. provides important new evidence confirming the significant and growing burden of HCC incidence, mortality, and health care cost among U.S. Medicare recipients, as well as the increasing contribution of the NAFLD etiology. The authors highlight novel observations including a larger number of HCC cases among younger Medicare enrollees under age 65 years, rapidly growing rates of $\mathrm{HCV}$-related $\mathrm{HCC}$ among Black Americans and NAFLD-related HCC among Hispanic Americans, and sharp rises in health resource utilization among Medicare recipients with HCC in both the inpatient and outpatient settings. This study has important limitations which are predominantly related to intrinsic characteristics of Medicare data, including reliance on flawed ICD-9 diagnosis codes, narrow study population not generalizable to non-Medicare patient cohorts, and limited information on key clinical variables which impact the outcomes of interest, including HCC stage, treatment, and liver transplantation. Future studies are especially needed to further characterize the unique pathogenesis, epidemiology, treatment, and health outcomes of HCC secondary to NAFLD, which now represents among the most important sources of liver-related morbidity and mortality in both Medicare and non-Medicare populations. 


\section{Acknowledgments}

None.

\section{Footnote}

Conflicts of Interest: The authors have no conflicts of interest to declare.

\section{References}

1. Jemal A, Ward EM, Johnson CJ, et al. Annual Report to the Nation on the Status of Cancer, 1975-2014, featuring Survival. J Natl Cancer Inst 2017. doi: 10.1093/jnci/ djx030.

2. Ryerson AB, Eheman CR, Altekruse SF, et al. Annual Report to the Nation on the Status of Cancer, 1975-2012, featuring the increasing incidence of liver cancer. Cancer 2016;122:1312-37.

3. Njei B, Rotman Y, Ditah I, et al. Emerging trends in hepatocellular carcinoma incidence and mortality. Hepatology 2015;61:191-9.

4. Centers for Disease Control and Prevention: National Center for Health Statistics. Chronic liver disease and cirrhosis death rates among persons aged 25 and over, by sex and age: United States, 2006-2016. Available online: https://www.cdc.gov/nchs/hus/contents2017. htm\#Figure_029

5. Asahina Y, Tsuchiya K, Tamaki N, et al. Effect of Aging on Risk for Hepatocellular Carcinoma in Chronic Hepatitis C Virus Infection. Hepatology 2010;52:518-27.

6. The Medicare Payment Advisory Commission. A Data Book: Health Care Spending and the Medicare Program. Section 2: Medicare beneficiary demographics. Available online: http://medpac.gov/docs/default-source/data-book/ jun18_databookentirereport_sec.pdf?sfvrsn=0

Cite this article as: Wong CR, Lim JK. Increasing contribution of non-alcoholic fatty liver disease to hepatocellular carcinoma incidence and mortality in U.S. Medicare. HepatoBiliary Surg Nutr 2019;8(5):506-508. doi: 10.21037/hbsn.2019.04.06
7. Hester D, Golabi P, Paik J, et al. Among Medicare patients with hepatocellular carcinoma, non-alcoholic fatty liver disease is the most common etiology and cause of mortality. J Clin Gastroenterol 2019. [Epub ahead of print].

8. Tapper EB, Parikh ND. Mortality due to cirrhosis and liver cancer in the United States , 1999-2016: observational study. BMJ 2018;362:k2817.

9. Kanwal F, Kramer JR, El-Serag H, et al. Race and Gender Differences in the Use of Direct Acting Antiviral Agents for Hepatitis C Virus. Clin Infect Dis 2016;63:291-9.

10. Chinchilla-López P, Ramírez-Pérez O, Cruz-Ramón V, et al. More Evidence for the Genetic Susceptibility of Medican Population to Nonalcoholic Fatty Liver Disease through PNPLA3. Ann Hepatol 2018;17:250-5.

11. Rinella ME. Nonalcoholic fatty liver disease: a systematic review. JAMA 2015;313:2263-73.

12. Younossi Z, Stepanova M, Ong JP, et al. Nonalcoholic steatohepatitis is the fastest growing cause of hepatocellular carcinoma in liver transplant candidates. Clin Gastroenterol Hepatol 2019;17:748-55.e3.

13. The Medicare Payment Advisory Commission. Report to the Congress: Medicare and the Health Care Delivery System. Chapter 3: Hospital inpatient and outpatient services. Available online: http://www.medpac.gov/docs/ default-source/reports/mar17_entirereport224610adfa9c6 65e80adff00009edf9c.pdf?sfvrsn=0

14. Wong CR, Njei B, Nguyen MH, et al. Survival after treatment with curative intent for hepatocellular carcinoma among patients with vs. without non-alcoholic fatty liver disease. Aliment Pharmacol Ther 2017;46:1061-9.

15. Younossi ZM, Otgonsuren M, Henry L, et al. Association of nonalcoholic fatty liver disease (NAFLD) with hepatocellular carcinoma (HCC) in the United States from 2004 to 2009. Hepatology 2015;62:1723-30. 